EESTI NSV TEADUSTE AKADEEMIA TOIMETISED. 28. KOIDE FOUSIKA * MATEMAATIKA. 1979, NR. 1

ИЗВЕСТИЯ АҚАДЕМИИ НАУК ЭСТОНСКОП ССР. ТОМ 28 ФИЗИКА * МАТЕМАТИКА. 1979, № 1

удК $530.12: 531.18$

П. КАРД

\title{
О РЕЛЯТИВИСТСКОМ ПОНЯТИИ МАССЫ
}

\section{Введение}

Понятие релятивистской массы включает, как известно, массу света. Одним из распространенных методов обоснования массы света является мысленный опыт Әйнштейна $\left[{ }^{1,2}\right]$ с закрытым цилиндром, смещающимся вдоль своей оси под действием излучаемой в одном конце и поглощаемой в другом конце световой вспышки. Если потребовать, что положение центра масс измениться не может, то следует заключить, что масса излучившего свет тела уменьшилась, а масса поглотившего тот же свет тела увеличилась на одну и ту же величину, равную импульсу света, деленному на его скорость $c$. Отсюда и вытекает, что свет переносит массу, в силу чего массу следует приписать самому свету. Притом, так как импульс света равен его энергии, деленной на $c$, масса оказывается равной энергии, деленной на $c^{2}$. Это - частный случай общего закона эквивалентности массы и энергии.

Несмотря на видимую простоту и подкупающую ясность этого мысленного опыта, ему присущи два недостатка. Первый состоит в нестрогости. Цилиндру приписывается абсолютная жесткость, что нереально. Как показано в $\left[{ }^{3}\right]$, попытка возможно полного описания этого опыта приводит к парадоксальному выводу о независимости массы тела от скорости. Но отказ от предположения об абсолютной жесткости цилиндра лишает опыт эвристической ценности. Это обстоятельство побудило В. Н. Стрельцова $[4,5]$ видоизменить опыт Эйнштейна. Он помещает излучающее тело не у самого основания цилиндра, а на некотором расстоянии от него с таким расчетом, чтобы оно, излучив световую вспышку, достигало основания цилиндра одновременно с поглощением света в другом теле у другого основания. В этом случае, действительно, абсолютная жесткость цилиндра не требуется и никаких трудностей не возникает. Легко видеть, однако, что и сам цилиндр в этой модификации опыта тоже не нужен. Достаточно рассматривать два тела, из которых одно излучает в направлении другого световую вспышку, а то ее поглощает. Ход рассуждений таков. Пусть два одинаковых тела покоятся вначале на оси $x$ в точках $x_{1}$ и $x_{2}\left(x_{1}<x_{2}\right)$, так что центр масс лежит в точке

$$
x=(1 / 2)\left(x_{1}+x_{2}\right) \text {. }
$$

Первое испускает в направлении второго световую вспышку с импульсом $p$ и начинает двигаться со скоростью $-p / m_{1}$, где $m_{1}$ - его релятивистская масса после испускания. За время $\left(x_{2}-x_{1}\right) / c$, потребное для достижения светом второго тела, первое передвинется на расстояние $\left(x_{2}-x_{1}\right) p / m_{1} c$ и в момент поглощения света вторым телом будет на- 
ходиться в точке $x_{1}-\left(x_{2}-x_{1}\right) p / m_{1} c$. Второе тело, поглотив свет, получит скорость $p / m_{2}$, где $m_{2}$ - его релятивистская масса после поглощения. Следовательно, по прошествии произвольного времени $t$ после поглощения координаты тел будут

$$
\begin{aligned}
& X_{1}=x_{1}-\frac{\left(x_{2}-x_{1}\right) p}{m_{1} c}-\frac{p t}{m_{1}}, \\
& X_{2}=x_{2}+\frac{p t}{m_{2}} .
\end{aligned}
$$

Координата центра масс в момент $t$ равна

$$
X=\frac{m_{1} X_{1}+m_{2} X_{2}}{m_{1}+m_{2}} ;
$$

подставляя сюда выражения (2), находим

$$
X=\frac{m_{1} x_{1}+m_{2} x_{2}-(p / c)\left(x_{2}-x_{1}\right)}{m_{1}+m_{2}} .
$$

Остается приравнять выражения (1) и (4):

$$
\frac{x_{1}+x_{2}}{2}=\frac{m_{1} x_{1}+m_{2} x_{2}-(p / c)\left(x_{2}-x_{1}\right)}{m_{1}+m_{2}},
$$

откуда

$$
m_{2}-m_{1}=2 p / c .
$$

Этот результат показывает, что свет перенес от первого тела ко второму массу $p / c$, которую и следует толковать как массу света.

Можно даже, убрав второе тело, еще более упростить схему опыта. Так поступает автор статьи $\left[{ }^{6}\right]$, где ход мыслей примерно таков. Тело с начальной массой $m$, покоясь в точке $x$, испускает в начальный момент световую вспышку с импульсом $p$, получая само скорость $-p / m_{1}$, где $m_{1}$ - его релятивистская масса после испускания. В момент $t$ оно находится в точке $x-p t / m_{1}$, а свет - в точке $x+c t$. Чтобы центр масс оставался в точке $x$, свету необходимо приписать массу. Обозначим ее через $\mu$. Тогда

$$
m_{1}\left(x-p t / m_{1}\right)+\mu(x+c t)=m x .
$$

Так как это равенство должно выполняться в любой момент, то

$$
p=\mu c
$$

H

$$
m_{1}+\mu=m
$$

Приведенные вариации мысленного опыта Әйнштейна показывают, что от цилиндра можно полностью избавиться. Тем самым исчезают и связанные с ним трудности. Но мысленному опыту Әйнштейна присущ еще другой недостаток. Он состоит в следующем. Қак уже отмечено в $\left[{ }^{3}\right]$, неизменность положения центра масс не есть первичный закон механики, а есть следствие законов сохранения массы и импульса. А так как импульс равен произведению массы на скорость, то требование сохранения положения центра масс неявно уже включает в себя ут- 
верждение, что свет с импульсом р имеет массу, равную $p / c$. Таким образом, в мысленном опыте Эйнштейна скрыт логический круг. То, что хотят доказать, уже содержится в исходной предпосылке - неизменности положения центра масс. Это значит, что опыт превращается всего лишь в иллюстрацию законов сохранения массы и импульса, а независимой доказательной силы не имеет. Особенно ясно это обнаруживается в последнем приведенном нами варианте опыта: чтобы написать формулу (7), мы должны были заранее потребовать наличия массы у света - иначе центр масс не был бы неподвижен. Следовательно, формула (8), полученная как вывод из формулы (7), фактически предшествует ей. Сохранение массы (формула (9)) тоже подразумевается в формуле (7). Мы приходим к выводу, что существование массы света есть прямое следствие законов сохранения массы и импульса, не нуждающееся ни в каком дополнительном мысленном опыте.

Все-таки мы не считаем вопрос на этом исчерпанным. Дело в том, что в литературе нередко масса света отрицается, а сохранение массы толкуется превратно (см., напр., $\left[{ }^{7,8}\right]$ ). При этом, конечно, приносится в жертву и неизменность положения центра масс. Согласиться с подобными воззрениями невозможно, а поэтому представляется методически и методологически важным способ, в котором закон сохранения массы не использовался бы в качестве исходной предпосылки, но получался бы как следствие из более ограниченного круга предпосылок. Притом полная сохраняющаяся масса должна включать массу света. Такой способ и будет изложен в настоящей статье.

\section{Масса света}

Будем исходить из следующих предпосылок: 1) принципа относительности; 2) релятивистской формулы преобразования скорости; 3) существования импульса света; 4) параллельности импульса любого объекта его скорости; 5) закона сохранения импульса в любой инерциальной системе. Покажем, что из этих предпосылок вытекает: 1) формула зависимости массы тела от скорости; 2) существование массы света; 3) релятивистские формулы преобразования массы и импульса; 4) закон сохранения массы в любой инерциальной системе.

Рассмотрим неподвижное тело с массой покоя $m_{0}$, излучающее световой поток со скоростью с и импульсом р. Так как эти векторы параллельны, то

$$
\mathrm{p}=\mu \mathrm{c}
$$

где $\mu$ - положительный скаляр с размерностью массы. Является ли $\mu$ на самом деле массой, пока неизвестно. Скорость тела после излучения обозначим через $\mathbf{u}$, а массу покоя его через $m_{1}$. Введем множитель $\gamma(u)$, определяющй зависимость массы от скорости, так что $m_{1} \gamma(u)$ есть масса тела, а $m_{1} \gamma(u) \mathbf{u}$ - его импульс. Сохранение импульса выражается в виде

$$
\mu \mathrm{c}+m_{1} \gamma(u) \mathbf{u}=0 .
$$

Перейдем в другую (штрихованную) инерциальную систему, движущуюся относительно предыдущей (нештрихованной) с произвольной скоростью v. Согласно общей формуле преобразования скорости, скорость излученного света в этой системе равна

$$
\mathbf{c}^{\prime}=\frac{\mathbf{c} \sqrt{1-v^{2} / c^{2}}+v^{-2}\left(1-\sqrt{\left.1-v^{2} / c^{2}\right)} \mathbf{c v} \cdot \mathbf{v}-\mathbf{v}\right.}{1-\mathrm{cv} / c^{2}}
$$


а импульс его обозначим как

$$
\mathrm{p}^{\prime}=\mu^{\prime} \mathbf{c}^{\prime}
$$

Скорость тела до излучения равна в штрихованной системе $-\mathbf{v}$, а после

$$
\mathbf{u}^{\prime}=\frac{\mathbf{u} \sqrt{1-v^{2} / c^{2}}+v^{-2}\left(1-\sqrt{1-v^{2} / c^{2}}\right)}{\mathbf{u v} \cdot \mathbf{v}-\mathbf{v}} .
$$

Соответственно, импульс тела равен до излучения света $-m_{0} \gamma(v) \mathrm{v}$, а после $m_{1} \gamma\left(u^{\prime}\right) \mathbf{u}^{\prime}$. Сохранение импульса выражается в виде

$$
-m_{0} \gamma(v) \mathbf{v}=m_{1} \gamma\left(u^{\prime}\right) \mathbf{u}^{\prime}+\mu^{\prime} \mathbf{c}^{\prime} .
$$

Теперь исключим из формул (11), (12), (14) и (15) векторы с, с' и и'. Сперва из формулы (11) имеем

$$
\mathbf{c}=-\frac{m_{1} \gamma(u) \mathbf{u}}{\mu} ;
$$

подставляя это выражение в формулу (12), находим

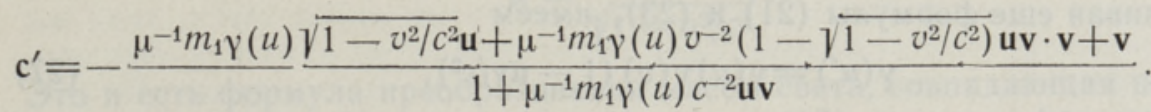

Затем подставляем выражения (14) и (17) в формулу (15). Результат таков:

$$
\begin{gathered}
m_{0} \gamma(v) \mathbf{v}+ \\
+\frac{m_{1} \gamma\left(u^{\prime}\right) \sqrt{1-v^{2} / c^{2}} \mathbf{u}+m_{1} \gamma\left(u^{\prime}\right) v^{-2}\left(1-\sqrt{\left.1-v^{2} / c^{2}\right)} \mathbf{u v} \cdot \mathbf{v}-m_{1} \gamma\left(u^{\prime}\right) \mathbf{v}\right.}{1-\mathbf{u v} / c^{2}}- \\
-\frac{\mu^{-1} \mu^{\prime} m_{1} \gamma(u) \sqrt{1-v^{2} / c^{2}} \mathbf{u}+\mu^{-1} \mu^{\prime} m_{1} \gamma(u) v^{-2}}{1+\mu^{-1} m_{1} \gamma(u) c^{-2} \mathbf{u v}}=0 .
\end{gathered}
$$

В этой формуле векторы $\mathbf{u}$ и $\mathbf{v}$ независимы. Следовательно, коэффициенты при $\mathbf{u}$ и $\mathbf{v}$ должны обращаться в нуль. Получаем два равенства

$$
\begin{gathered}
m_{0} \gamma(v)+\frac{m_{1} \gamma\left(u^{\prime}\right) v^{-2}\left(1-\sqrt{\left.1-v^{2} / c^{2}\right)} \mathbf{u v}-m_{1} \gamma\left(u^{\prime}\right)\right.}{1-\mathbf{u v} / c^{2}}- \\
-\frac{\mu^{-1} \mu^{\prime} m_{1} \gamma(u) v^{-2}\left(1-\sqrt{1-v^{2} / c^{2}}\right) \mathbf{u v}+\mu^{\prime}}{1+\mu^{-1} m_{1} \gamma(u) c^{-2} \mathbf{u v}}=0
\end{gathered}
$$

и

$$
\frac{\mu \gamma\left(u^{\prime}\right)}{1-\mathbf{u v} / c^{2}}=\frac{\mu^{\prime} \gamma(u)}{1+\mu^{-1} m_{1} \gamma(u) c^{-2} \mathbf{u v}},
$$

образующих систему уравнений для $\mu$ и $\mu^{\prime}$. Решая ее, находим

$$
\mu=-m_{1} \gamma(u)+\frac{m_{0 \gamma}(u) \gamma(v)\left(1-\mathbf{u v} / c^{2}\right)}{\gamma\left(u^{\prime}\right)}
$$




$$
\mu^{\prime}=-m_{1} \gamma\left(u^{\prime}\right)+m_{0} \gamma(v) .
$$

Здесь обратим внимание на то, цто $\mu$ не может зависеть от $\mathbf{v}$. Формула (21) должна быть верна при любом значении $\mathbf{v}$. Положив $\mathbf{v}=0$, так что $\gamma(v)=1$ и $\mathbf{u}^{\prime}=\mathbf{u}$, находим

$$
\mu=-m_{1} \gamma(u)+m_{0} .
$$

А если положим $\mathbf{v}=\mathbf{u}$, так что $\mathbf{u}^{\prime}=0, \gamma\left(u^{\prime}\right)=1$ и $\gamma(v)=\gamma(u)$, то получим

$$
\mu=-m_{1} \gamma(u)+m_{0} \gamma^{2}(u)\left(1-u^{2} / c^{2}\right) .
$$

Сравнивая эту формулу с предыдущей, находим

$$
\gamma(u)=\left(1-u^{2} / c^{2}\right)^{-1 / 2} .
$$

Итак, мы нашли формулу зависимости массы тела от скорости:

$$
m(u)=\frac{m_{0}}{\sqrt{1-u^{2} / c^{2}}} .
$$

Сравнивая еще формулы (21) и (23), имеем

$$
\gamma\left(u^{\prime}\right)=\gamma(u) \gamma(v)\left(1-\mathbf{u v} / c^{2}\right),
$$

или, согласно формуле (25),

$$
\sqrt{1-u^{\prime 2} / c^{2}}=\frac{\sqrt{1-u^{2} / c^{2}} \sqrt{1-v^{2} / c^{2}}}{1-\mathbf{u v} / c^{2}}
$$

Эта формула получается также прямо из формулы преобразования скорости (14).

Вернемся к формулам (22) и (23). В нештрихованной инерциальной системе $m_{0}$ - начальная, $m_{1} \gamma(u)$ - конечная масса тела. Согласно формуле (23), масса уменьшилась на $\mu$. В штрихованной системе, аналогично, $m_{0} \gamma(v)$ - начальная масса тела, $m_{1} \gamma\left(u^{\prime}\right)$ - конечная; формула (22) показывает, что масса уменьшилась на $\mu^{\prime}$. Если бы мы таким же образом рассмотрели процесс поглощения телом светового потока, то нашли бы, что в любой инерциальной системе масса тела увеличивается на величину, равную импульсу света, деленному на его скорость. Итак, мы обнаружили сохраняющуюся величину. Каждый объект, будь то тело или световой поток, характеризуется величиной, равной деленному на скорость импульсу. Сохраняется, и притом в любой инерциальной системе, сумма значений этой величины, взятых для всех участвующих в процессе объектов. У тел эта величина называется издавна массой. Естественно распространить то же название и на свет. Итак, мы приходим к фундаментальному выводу о том, что свет является носителем не только импульса, но и массы. Кроме того, мы убедились, что при излучении и поглощении света телом полная масса сохраняется.

\section{Формулы преобразования массы и импульса и общий закон сохранения массы}

Для массы и импульса тела формулы преобразования получаются очень просто из формул (14), (26) и (28). Сначала из формул (26) и (28) имеем 


$$
m^{\prime}=\frac{m-\mathbf{p v} / c^{2}}{\sqrt{1-v^{2} / c^{2}}}
$$

(здесь $m=m(u)$ и $\left.m^{\prime}=m\left(u^{\prime}\right)\right)$. Это - формула преобразования массы. Умножая ее на формулу (14), находим формулу преобразования импульса:

$$
\mathbf{p}^{\prime}=\frac{\mathbf{p} \sqrt{1-v^{2} / c^{2}}+\left(\mathbf{p v} \cdot \mathbf{v} / v^{2}\right)\left(1-\sqrt{\left.1-v^{2} / c^{2}\right)}-m \mathbf{v}\right.}{\sqrt{1-v^{2} / c^{2}}}
$$

Для массы и импульса света формулы преобразования точно такого же вида получаются из формул (16), (22), (23) и (27). Сперва из формулы (22) с учетом формулы (27) следует

$$
\mu^{\prime}=\gamma(v)\left[-m_{1} \gamma(u)\left(1-\mathbf{u v} / c^{2}\right)+m_{0}\right] .
$$

Исключая отсюда с помощью формул (16) и (23) $m_{0}$ и $m_{1}$ и заменяя $\mu \mathrm{c}$ на $\mathrm{p}$, находим:

$$
\mu^{\prime}=\frac{\mu-\mathrm{pv} / c^{2}}{\sqrt{1-v^{2} / c^{2}}}
$$

Это и есть формула преобразования массы света, совпадающая по виду с формулой (29). Умножив ее на формулу (12), находим формулу преобразования импульса света

$$
\mathbf{p}^{\prime}=\frac{\mathrm{p} \sqrt{1-v^{2} / c^{2}}+\left(\mathbf{p v} \cdot \mathbf{v} / v^{2}\right)\left(1-\sqrt{1-v^{2} / c^{2}}\right)-\mu \mathbf{v}}{\sqrt{1-v^{2} / c^{2}}}
$$

совпадающую по виду с формулой (30).

Обратимся к вопросу о сохранении массы. В предыдущем разделе мы видели, что масса при поглощении и испускани! света сохраняется. Теперь легко с помощью формул преобразования обобщить закон сохранения массы на любые процессы. Из линейности формул преобразования (29) и (30) или (32) и (33) вытекает, что полный импульс Р и полная масса $M$ любой совокупности объектов преобразуются по формулам того же внда, т. е.

$$
\begin{aligned}
& \mathbf{P}^{\prime}=a_{11} \mathbf{P}+\mathbf{a}_{12} M, \\
& M^{\prime}=a_{22} M+\mathbf{a}_{21} \mathbf{P},
\end{aligned}
$$

где $a_{11}, a_{22}, \mathbf{a}_{12}$ и $\mathbf{a}_{21}$ - коэффициенты, зависящие от относительной скорости $\mathbf{v}$ двух инерциальных систем. Из первой формулы (34) следует, что если $\mathbf{P}$ и $\mathbf{P}^{\prime}$ сохраняются, то сохраняется и $M$, а из второй видно, что если сохраняются $\mathbf{P}$ и $M$, то сохраняется и $M^{\prime}$. Итак, общий закон сохранения импульса имеет следствием столь же общий закон сохранения массы.

\section{Заключйтельные замечания}

Изложенный в настоящей статье метод обоснования понятия массы света и общего релятивистского понятия массы как сохраняющейся величины свободен от недостатков метода цилиндра Эйнштейна и предпочтителен также в том отношении, что в нем используется меньшее 
число предпосылок. Часто в литературе, где релятивистская динамика строится на основе законов сохранения, сохранение массы принимается в качестве независимой предпосылки наряду с сохранением импульса. Правда, М. Борн в [ $\left.{ }^{9}\right]$, рассматривая при выводе зависимости массы тела от скорости неупругое соударение тел, показывает, как сохранение массы вытекает из сохранения импульса (с. $323-330)$. Однако вывод М. Борна идет мимо понятия массы света, для обоснования которого он привлекает мысленный опыт Эйнштейна с цилиндром (с. 342-345). Преимущество нашего метода мы видим не только в возможности отказаться от этого опыта, но и в большей компактности и цельности.

Еще одно замечание таково. Говоря о массе света, мы ничего не сказали о его энергии. Конечно, если свет с импульсом р имеет массу $\mu=p / c$, то его энергия $E$ оказывается связанной с массой равенством $E=\mu c^{2}$ (так как $\left.p=E / c\right)$. Но этот факт относится совсем к другому вопросу - к закону эквивалентности массы и энергии. Хотя масса света играет важную роль в правильном истолковании этого закона, она одна не дает его полного обоснования. Поэтому доказательство существования массы света не следует понимать всего лишь как доказательство «инерции энергии», как говорил еще А. Эйнштейн и как говорится иногда по традиции и ныне. Доказывая существование массы света, мы доказываем в первую очередь инертность света, что имеет точный смысл независимо от энергии. Подчеркнем, что понятие массы света, хотя мы обосновывали его с релятивистской точки зрения, не является специфически релятивистским, поскольку оно поддается обоснованию и в рамках нерелятивистской физики (см. $\left.\left[{ }^{10,11}\right]\right)$. Наоборот, закон эквивалентности массы и энергии, не имея в нерелятивистской физике никакого аналюга, является полностью релятивистским (см. по этому поводу $\left[{ }^{12}\right]$ ). Вот почему мы разделяем эти два вопроса.

\section{ЛИТЕРАТУ РА}

1. E inste in, A., Ann. Phys., 20, № 8, 627-633 (1906).

2. Э й нш т е й н А., Собрание научных трудов, т. I, М., «Наука», 1965, с. 39.

3. К а р д П., Изв. АН ЭССР, Физ. Матем., 24, № 2, 173-177 (1975).

4. С трельцов В. Н., К одному элементарному выводу соотношения $E=m c^{2}$, ОИЯИ, Дубна, 1968, Р2-3672.

5. С трельцов В. Н., Некоторые вопросы специальной теории относительности. (Релятивистская электродинамика), ОИЯИ, Дубна, 1977, Р2-11115, с. 15.

6. A n t i p p a, Adel F., Amer. J. Phys., 44, № 9, $841-844$ (1976).

7. У г а ров В. А., Специальная теория относительности, изд. 2-е, М., «Наука», 1977.

8. Т е р лецкий Я. П., В кн.: История и методология естественных наук, вып. 15, М., Изд-во Моск. ун-та, 1974, с. $3-15$.

9. Б о р н М., Эйнштейнов́ская теория относительности, М., «Мир», 1964.

10. К а р д П., Изв. АН ЭССР, Физ. Матем., 24, № 3, 305-311 (1975).

11. К а р д П., Изв. АН ЭССР, Физ. Матем., 25, № 1, 15-22 (1976).

12. К а р д П. Г., Уч. зап. Тартуск. гос. ун-та, вып. 360, 21-28 (1975).

Тартуский государственный университет
Поступила в редакцию 20/VI 1978 


\section{P. KARD}

\section{RELATIVISTLIKU MASSI MÕISTEST}

Relativistliku massi mõiste hõlmab teatavasti valguse massi. Seda põhjendatakse sageli Einsteini mõttelise katsega $\left[{ }^{1,2}\right]$, mis aga päris range ei ole $\left[{ }^{3}\right]$. Tegelikult peitub temas loogikaring, sest katse tôlgendamisel eeldatakse varjatult juba ette valguse massi olemasolu. Artiklis on tõestatud seevastu nii valguse massi olemasolu kui ka massi jäävus, eeldades ainult impulsi olemasolu valgusel, impulsi jäävust, impulsi paralleelsust kiirusega ja kiiruse relativistlikku teisendusvalemit. Selleks on vaadeldud kindlasuunalise valgusvoo kiirgumist kehast kahes inertsiaalsüsteemis - keha algse paigaloleku süsteemis ja selle suhtes meelevaldse kiirusega v liikuvas süsteemis. Impulsi jäävust väljendavad seosed (11) ja (15), kus $m_{0}$ on keha seisumass enne ja $m_{1}$ pärast kiirgamist, $\gamma-$ tegur, mille poolest liikuva keha mass erineb seisumassist, $\mathbf{u}, \mathbf{c}, \mu \mathbf{c}$ ning $\mathbf{u}^{\prime}, \mathbf{c}^{\prime}, \mu^{\prime} \mathbf{c}^{\prime}-$ keha ja valgusvoo kiirused ja valgusvoo impulss kahes inertsiaalsüsteemis. Kiiruste $\mathbf{c}, \mathbf{c}^{\prime}$ ja $\mathbf{u}^{\prime}$ elimineerimine nendest seostest ning kiiruste c ja u teisendusvalemitest (12) ja (14) annab seose (18), millest vektorite $\mathbf{u}$ ja $\mathbf{v}$ sõltumatuse tõttu järelduvad seosed (21) ja (22). Siit järgnevad $\mu$ sõltumatuse tôttu $v$-st seosed (23), (26) ja (28). Seostest (22) ja (23) ilmneb, et mōlemas inertsiaalsüsteemis on vaadeldava protsessi puhul impulsi jagatis kiirusega jääv. Keha puhul võrdub see jagatis massiga. Jäävuse tōttu rakendub sama mõiste ka valgusele. Massi jäävuse üldine seadus järeldub massi ja impulsi teisendusvalemitest (29) ja (30) (keha puhul) või (32) ja (33) (valguse puhul), mis omakorda on valemite (14), (26) ja (28) või (11), (22), (23) ja (28) järeldus.

\section{P. KARD}

\section{UBER DEN RELATIVISTISCHEN MASSENBEGRIFF}

Der relativistische Massenbegriff bezieht bekanntlich die Masse des Lichtes ein. Als eine Begründung dafür gilt das Gedankenexperiment Einsteins $\left[{ }^{1,2}\right]$, das aber nicht ganz streng ist [3]. In der Tat birgt es einen ZirkelschluB in sich, insofern als bei der Deutung desselben die Existenz der Masse des Lichtes schon im voraus stillschweigend angenommen wird. Im Aufsatze hingegen beweist man die Existenz der Masse des Lichtes und die Erhaltung der Masse ausgehend nur von der Erhaltung des Impulses und der Existenz des Impulses des Lichtes, wobei auch die Parallelität des Impulses und der Geschwindigkeit und die relativistische Transformationsformel für Geschwindigkeit berücksichtigt werden. Dazu benutzt man die Ausstrahlung eines wohlgerichteten Lichtbündels von einem vorerst ruhenden Körper. Denselben Vorgang betrachtet man auch in einem anderen Inertialsystem, das sich mit einer beliebigen Geschwindigkeit v bewegt. Die Erhaltung des Impulses geben in beiden Systemen die Formeln (11) und (15) an, wo $m_{0}$ und $m_{1}$ die Ruhmasse des Körpers vor und nach der Ausstrahlung sind, $\gamma$ - ein geschwindigkeitsabhängiger Massenfaktor, $\mathbf{u}, \mathbf{u}^{\prime}$ - die Geschwindigkeit des Körpers nach der Ausstrahlung, $\mathbf{c}, \mathbf{c}^{\prime}$ und $\mu \mathbf{c}, \mu^{\prime} \mathbf{c}^{\prime}-$ die Geschwindigkeit und der Impuls des ausgestrahlten Lichtes in beiden Systemen. $\mathbf{c}^{\prime}$ und $\mathbf{u}^{\prime}$ werden durch die Transiormationsformeln (12) und (14) ausgedrückt. Die Elimination von $\mathbf{c}, \mathbf{c}^{\prime}$ und $\mathbf{u}^{\prime}$ aus den genannten vier Gleichungen ergibt die Gleichung (18), aus der man infolge der Unabhängigkeit von $\mathbf{u}$ und $\mathbf{v}$ die Gleichungen (21) und (22) schließt. Aus (21), $\mathrm{da}$ auch $\mu$ von $\mathbf{v}$ unabhängig ist, folgen weitere Gleichungen (23), (26) und (28). Die Gleichungen (22) und (23) lehren, daß in beiden Inertialsystemen der Quotient des Impulses durch die Geschwindigkeit erhalten bleibt. Beim Körper nennt man diesen Quotient von jeher Masse. Der eben festgestellte Erhaltungssatz legt es nahe, denselben Begriff auf das Licht zu erweitern. Allgemein ist der Erhaltungssatz der Masse eine Folge der Transformationsformeln für Masse und Impuls des Körpers (Formeln (29) und (30)) oder des Lichtes (Formeln (32) und (33)), die ihrerseits aus den Formeln (14), (26) und (28) resp. (11), (22), (23) und (28) gefolgert werden können. 